\title{
Hilbert Space Theoretic Methods in the Study of Correlation Measures
}

\author{
Sanford I. Levy \\ Department of Mathematics, Indiana University, Bloomington, Indiana, USA
}

Received November 14, 1973

\begin{abstract}
We study the uniqueness of the association of a classical statistical mechanical state and its sequence of modified correlation measures by studying a cyclic representation of the canonically associated Börchers algebra of the system. We show that semi-analyticity of the cyclic vector for a particular family of symmetric operators is sufficient for uniqueness while analyticity of the cyclic vector permits us to recover the state from the modified correlation measures.
\end{abstract}

\section{§ 1. Introduction}

The problem of the unique specification of a state of a classical statistical mechanical system by an infinite sequence of correlation functions has been studied, in the past, within two widely differing mathematical frameworks. The first is the algebraic approach as typified by the work of Ruelle Ref. [1], and the second is the measure theoretic approach due to Lenard Ref. [2]. In this paper the two approaches are combined.

From the outset we assume the structure, notation, and results of Ref. [2]. Thus the state of a system is represented by a probability measure on the space of locally finite configurations, $X$, constructed from the one particle space, $(E, \mathscr{R})$, where $\mathscr{R}$ is a ring of subsets of $E$. We study not correlation functions but correlation measures, the $n^{\text {th }}$ correlation measure, $\varrho_{n}$, being a measure on $\left(E^{n}, \Sigma\left[\mathscr{R}^{n}\right]\right)$ where $\Sigma\left[\mathscr{R}^{n}\right]$ is the $\sigma$-algebra generated by $\mathscr{R}^{n}$.

We construct a "Börchers algebra" from this framework and obtain a representation of it. This is done by introducing "modified correlation measures" which define a "state" on the algebra, i.e. a positive linear functional, and then applying the Gelfand-Naimark-Segel construction. The representation so obtained is cyclic and the operators commute on a dense invariant subspace.

We show that semi-analyticity of the cyclic vector for the operator representatives of the Börchers algebra is sufficient to guarantee uniqueness of the probability measure defining the correlation measures. 
Additionally, by strengthening the condition on the cyclic vector to analyticity we obtain essential self-adjointness of certain symmetric elements of the operator algebra and then an expression for the probability measure in terms of the spectral measures of the self-adjoint closures of these operators.

\section{$\S$ 2. Modified Correlation Measures}

We assume we have at our disposal a set $E$ and a ring of subsets of $E, \mathscr{R}$. From this we construct the measurable space

$$
(X, \Sigma[\mathbb{C}])
$$

where $X$ is the space of locally finite configurations introduced by Lenard and $\Sigma[\mathbb{C}]$ is the $\sigma$-algebra generated by the ring of sets $\mathbb{C}$ which is generated by the cylinder sets.

Let $\mu$ be a state on $X$, i.e. a probability measure on

$$
(X, \Sigma[\mathbb{C}])
$$

then the sequence of correlation measures is defined by

$$
\varrho_{n}(H)=\int_{X} N_{H} d \mu, \quad H \in \Sigma\left[\mathscr{R}^{n}\right],
$$

where, for $\xi \in X, \xi=\left(x_{1}, x_{2}, \ldots\right)$ an enumeration of $\xi$, $N_{H}(\xi)=$ the number of ordered $n$-tuples of distinct integers, $\left(i_{1}, \ldots, i_{n}\right)$, satisfying $\left(x_{i_{1}}, \ldots, x_{i_{n}}\right) \in H$.

We define the modified correlation measures, $\hat{\varrho}_{n} n=1,2, \ldots$, in an analogous fashion. For $H \in \Sigma\left[\mathscr{R}^{n}\right]$, and $\xi \in X$, with $\xi=\left(x_{1}, x_{2}, \ldots\right)$, let $\hat{N}_{H}(\xi)=$ the number of ways one can choose ordered $n$-tuples of integers

such that

$$
\left(i_{1}, i_{2}, \ldots, i_{n}\right)
$$

$$
\left(x_{i_{1}}, \ldots, x_{i_{n}}\right) \in H \text {. }
$$

Note that the word "distinct" is missing from this definition.

Fact. $\hat{N}_{H}$ is a measurable function. The proof of this is identical to the proof of the measurability of $N_{H}$ given in Ref. [2].

We define

$$
\hat{\varrho}_{n}(H):=\int_{X} \hat{N}_{H} d \mu .
$$

The motivation for the definition of $\hat{N}_{H}$ and $\hat{\varrho}_{n}$ is contained in Appendix 1. 


\section{§ 3. Construction of the Hilbert Space}

Definition. Let $\mathfrak{F}$ be a family of subsets of a set $F$. A simple function

$$
\zeta=\sum_{k=1}^{\ell} \alpha_{k} \chi_{M_{k}}, \quad M_{k} \subset F
$$

is said to be based on $\mathfrak{F}$ if

Let

$$
M_{k} \in \mathfrak{F}, \quad k=1,2, \ldots, \ell .
$$

$$
\mathfrak{C}_{n}=\left\{\Lambda_{1} \Lambda_{2} \ldots \Lambda_{n}: \Lambda_{i} \in \mathscr{R}\right\} .
$$

We denote by $\mathfrak{S}_{n}$ the linear space of simple functions defined on $E^{n}$ and based on $\mathfrak{C}_{n}$. Finally, let $\subseteq$ be the vector space direct sum of the $\mathfrak{S}_{n}$ :

$$
\Im=\underset{n=0}{\infty} \Xi_{n} .
$$

We adopt the usual convention that

$$
\Im_{0}=\mathbb{C} \text {. }
$$

$\Im$ is the linear space of sequences

with

$$
\left(\varphi_{n}\right)_{n=0}^{\infty}
$$

$$
\varphi_{n} \in \mathfrak{\Im}_{n}
$$

and $\varphi_{n}=0$ for all but a finite number of $n \in \mathbb{Z}_{+}$. The special case

$$
\varphi=\left(0,0, \ldots, \chi_{\Lambda_{1} \ldots \Lambda_{n}}, 0, \ldots\right)
$$

we denote by

$$
\varphi_{\Lambda_{1} \ldots A_{n}},
$$

We make $\subseteq$ into an algebra with involution in the usual way. Multiplication is defined by

$$
(\varphi \otimes \psi)_{n}\left(x_{1}, \ldots, x_{n}\right):=\sum_{k=0}^{k} \varphi_{k}\left(x_{1}, \ldots, x_{k}\right) \psi_{n-k}\left(x_{k+1}, \ldots, x_{n}\right)
$$

and involution by

$$
\left(\varphi^{*}\right)_{n}\left(x_{1}, \ldots, x_{n}\right):=\overline{\varphi_{n}\left(x_{n}, x_{n-1}, \ldots, x_{1}\right)} .
$$

It is not difficult to show that

and

$$
\otimes: \mathfrak{S} \times \mathbb{S} \rightarrow \mathfrak{S}
$$

$$
(\varphi \otimes \psi)^{*}=\psi^{*} \otimes \varphi^{*} ;
$$

the other linear operations and properties of involution being obvious we indeed have an algebra with involution, the Börchers algebra for the system. 
We now define a positive linear functional on $\mathbb{S}$ by means of the sequence of measures

For $\varphi=\left(\varphi_{n}\right)_{n=0}^{\infty} \in \mathbb{S}$

$$
\left(\varrho_{n}\right)_{n=1}^{\infty} \text {. }
$$

$$
\hat{\varrho}(\varphi):=\varphi_{0}+\sum_{n=1}^{\infty} \int_{E^{n}} \varphi_{n} d \hat{\varrho}_{n} .
$$

We note that this is always a finite sum. For convenience we shall write

$$
\hat{\varrho}(\varphi)=\sum_{n=0}^{\infty} \int_{E^{n}} \varphi_{n} d \hat{\varrho}_{n} .
$$

We now use the fact that $\left(\varrho_{n}\right)_{n=1}^{\infty}$ are modified correlation measures associated with

$$
(X, \Sigma[\mathfrak{C}], \mu)
$$

to obtain another expression for $\varrho(\varphi)$.

First:

$$
\begin{aligned}
\hat{\varrho}\left(\varphi_{\Lambda_{1} \ldots \Lambda_{n}}\right) & =\int_{E^{n}} \chi_{\Lambda_{1} \ldots \Lambda_{n}} d \varrho_{n} \\
& =\varrho_{n}\left(\Lambda_{1} \ldots \Lambda_{n}\right) \\
& =\int_{X} \hat{N}_{\Lambda_{1} \ldots \Lambda_{n}} d \mu .
\end{aligned}
$$

Define for $\varphi=\left(\varphi_{n}\right)_{n=0}^{\infty}$ and $\varphi_{n}=\sum_{k=1}^{l} \alpha_{k} \chi_{M_{k}}$ the following functions on $X$ :

and

$$
\hat{\eta}_{\varphi_{n}}:=\sum_{k=1}^{l} \alpha_{k} \hat{N}_{M_{k}}, \quad n=1,2, \ldots
$$

[For $\left.n=0, \hat{\eta}_{\varphi_{0}}=\varphi_{0} \circ \chi_{X} \cdot\right]$

$$
\hat{\eta}_{\varphi}:=\sum_{n=0}^{\infty} \hat{\eta}_{\varphi_{n}}
$$

Then, since everything in sight is linear,

$$
\hat{\varrho}(\varphi)=\int_{X} \hat{\eta}_{\varphi} d \mu \text {. }
$$

Proposition 3.1. $\varrho$ is a positive linear functional on $\mathfrak{\Xi}$.

Proof. Linearity is obvious. To show

$$
\hat{\varrho}\left(\varphi^{*} \otimes \varphi\right) \geqq 0
$$

we use formula (3.1) to show, in general,

Then

$$
\hat{\varrho}\left(\varphi^{*} \otimes \psi\right)=\int_{X} \overline{\hat{\eta}}_{\varphi} \hat{\eta}_{\psi} d \mu .
$$

$$
\hat{\varrho}\left(\varphi^{*} \otimes \varphi\right)=\int_{X}\left|\hat{\eta}_{\varphi}\right|^{2} d \mu \geqq 0 .
$$


We now apply the Gelfand-Naimark-Segal construction to the algebra $\subseteq$ with the positive linear functional $\varrho$. In this way we obtain

i) a Hilbert space, $\mathscr{H}$, in which $\mathfrak{S}$ is mapped onto a dense linear manifold, $\mathscr{D}$, and

ii) a representation of $\mathfrak{S}$ as an algebra of operators defined on $\mathscr{D}$ which is invariant for each operator.

We introduce the following notation:

For $\varphi \in \mathfrak{S}, \Psi_{\varphi}$ is its image in $\mathscr{D}$ and $A_{\varphi}$ is its representative as an operator. Denoting the unit element in $\mathbb{\varsigma}$ by

we let

$$
e \equiv(1,0,0, \ldots)
$$

$$
\Omega \equiv \Psi_{e} .
$$

By the G.N.S. construction $\Omega$ is a cyclic vector for the representation and

and

$$
A_{\varphi} \Psi_{\psi}=\Psi_{\varphi \otimes \psi}
$$

$$
\hat{\varrho}(\varphi)=\left(A_{\varphi} \Omega, \Omega\right) \text {. }
$$

Proposition 3.2. Let $\Lambda \in \mathscr{R}$. Then $A_{\varphi_{\Lambda}}$ is a positive [hence symmetric] operator.

Proof. For $\varphi \in \mathfrak{S}$

$$
\begin{aligned}
\left(A_{\varphi_{\Lambda}} \Psi_{\varphi}, \Psi_{\varphi}\right) & =\left(A_{\varphi_{\Lambda}} A_{\varphi} \Omega, A_{\varphi} \Omega\right) \\
& =\left(A_{\varphi}^{*} A_{\varphi_{\Lambda}} A_{\varphi} \Omega, \Omega\right) \\
& =\left(A_{\varphi^{*}} A_{\varphi_{\Lambda}} A_{\varphi} \Omega, \Omega\right)
\end{aligned}
$$

[since $\mathscr{D} \subset$ domain of $\left(A_{\varphi}\right)^{*}$ and $\left.\left.\left(A_{\varphi}\right)^{*}\right|_{\mathscr{D}}=A_{\varphi^{*}}\right]$.

Thus

$$
\begin{aligned}
\left(A_{\varphi_{\Lambda}} \Psi_{\varphi}, \Psi_{\varphi}\right) & =\left(A_{\varphi^{*} \otimes \varphi_{\Lambda} \otimes \varphi} \Omega, \Omega\right) \\
& =\varrho\left(\varphi^{*} \otimes \varphi_{\Lambda} \otimes \varphi\right) \\
& =\int_{X}\left|\hat{\eta}_{\varphi}\right|^{2} \hat{N}_{\Lambda} d \mu \geqq 0 .
\end{aligned}
$$

Proposition 3.3. Let $\varphi, \psi \in \mathbb{S}$. Then $A_{\varphi}$ and $A_{\psi}$ commute on $\mathscr{D}$.

Proof. Fix $\varphi^{\prime} \in \mathbb{S}$ and consider for arbitrary $\psi^{\prime} \in \mathbb{S}\left(A_{\varphi} A_{\psi} \Psi_{\varphi^{\prime}}, \Psi_{\psi^{\prime}}\right)$ and $\left(A_{\psi} A_{\varphi} \Psi_{\varphi^{\prime}}, \Psi_{\psi^{\prime}}\right)$. Performing the usual manipulations shows that each expression can be written as

Thus for $\Psi \in \mathscr{D}$

$$
\int_{X} d \mu \hat{\eta}_{\psi^{\prime *}} \hat{\eta}_{\varphi} \hat{\eta}_{\psi} \hat{\eta}_{\varphi^{\prime}}
$$

$$
\left(A_{\varphi} A_{\psi} \Psi_{\varphi^{\prime}}, \Psi\right)=\left(A_{\psi} A_{\varphi} \Psi_{\varphi^{\prime}}, \Psi\right) .
$$

The denseness of $\mathscr{D}$ and the Riesz representation theorem complete the proof. 
Thus we see that our patently non-commutative algebra has a representation as an algebra of operators which commute on a common invariant dense domain. This fact arises simply because all commutators in $\subseteq$ are in the kernel of $\hat{\varrho}$.

\section{§ 4. Semi-Analyticity of $\Omega$ and Uniqueness of the State}

First we recall that a vector, $\Psi$, in a Hilbert space is semi-analytic for a densely defined operator, $A$, in $\mathfrak{h}$ if

i) $\Psi \in \operatorname{Dom}\left(A^{n}\right) n=1,2, \ldots$ and

ii) the power series

$$
\sum_{n=0}^{\infty} \frac{\left\|A^{n} \Psi\right\|}{(2 n) !} z^{n}
$$

has a non-zero radius of convergence.

In this section we show that semi-analyticity of $\Omega$ for the symmetric operators

$$
A_{\varphi_{\Lambda}}, \quad \Lambda \in \mathscr{R}
$$

is sufficient to ensure uniqueness of the measure $\mu$ on $(X, \Sigma[\mathbb{C}])$ which defined the sequence of modified correlation measures

$$
\left(\varrho_{n}\right)_{n=1}^{\infty} \text {. }
$$

This is done by showing that the semi-analyticity condition on $\Omega$ implies a convergence condition [C.C.] on the modified correlation measures which is sufficient to ensure uniqueness.

For convenience we state the convergence condition we will arrive at.

C.C.: The power series

$$
\sum_{n=0}^{\infty} \frac{\hat{\varrho}\left(\Lambda^{n}\right)}{(2 n) !} z^{n}
$$

has a non-zero radius of convergence for any $\Lambda \in \mathscr{R}$. For convenience we drop the subscript $n$ from $\hat{\varrho}_{n}$ adopting the convention that for $\Lambda \in \mathscr{R} \hat{\varrho}\left(\Lambda^{n}\right) \equiv \hat{\varrho}_{n}\left(\Lambda^{n}\right)$.

Proposition 4.1. Let $\Omega$ be semi-analytic for

$$
A_{\varphi_{\Lambda}} \forall \Lambda \in \mathscr{R}
$$

then the sequence $\left(\varrho_{n}\right)_{n=1}^{\infty}$ satisfies C.C.

Proof. Since $\Omega$ is semi-analytic for $A_{\varphi_{A}}$ the series

$$
\sum_{n=0}^{\infty} \frac{\left\|A_{\varphi_{\Lambda}}^{n} \Omega\right\|}{(2 n) !} z^{n}
$$


has a non-zero radius of convergence. Note that

$$
\begin{aligned}
\left\|A_{\varphi_{\Lambda}}^{n} \Omega\right\|^{2} & =\left(A_{\varphi_{\Lambda}}^{n} \Omega, A_{\varphi_{A}}^{n} \Omega\right) \\
& =\left(A_{\varphi_{A}}^{2 n} \Omega, \Omega\right)=\hat{\varrho}\left(\varphi_{\Lambda} 2 n\right) \\
& =\int \chi_{\Lambda^{2 n}} d \hat{\varrho}_{2 n} \\
& =\hat{\varrho}\left(\Lambda^{2 n}\right) .
\end{aligned}
$$

Since the "square-root" is a concave function we apply Jensen's inequality to obtain

i.e.

$$
\hat{\varrho}\left(\Lambda^{n}\right)=\int_{X} \hat{N}_{\Lambda}^{n} d \mu \leqq\left[\int_{X} \hat{N}_{\Lambda}^{2 n} d \mu\right]^{\frac{1}{2}}=\hat{\varrho}\left(\Lambda^{2 n}\right)^{\frac{1}{2}} .
$$

$$
\hat{\varrho}\left(\Lambda^{n}\right) \leqq\left\|A_{\varphi_{\Lambda}}^{n} \Omega\right\| \text {. }
$$

We now show that C.C. implies the uniqueness of $\mu$ associated with $\left(\varrho_{n}\right)_{n=1}^{\infty}$.

Proposition 4.2. A necessary and sufficient condition for the power series

$$
\sum_{m_{1}, \ldots, m_{r}} \hat{\varrho}\left(\Lambda_{1}^{m_{1}} \ldots \Lambda_{r}^{m_{r}}\right) \frac{z_{1}^{m_{1}}}{\left(2 m_{1}\right) !} \cdots \frac{z_{r}^{m_{r}}}{\left(2 m_{r}\right) !}
$$

to have a non-zero radius of convergence for arbitrary

$$
\left(\Lambda_{1}, \ldots, \Lambda_{r}\right), \quad \Lambda_{r} \in \mathscr{R}, \quad \text { and } r,
$$

is that the power series

$$
\sum_{m=0}^{\infty} \hat{\varrho}\left(\Lambda^{m}\right) \frac{z^{m}}{(2 m) !}
$$

has a non-zero radius of convergence for any $\Lambda \in \mathscr{R}$.

Proof. Necessity is obvious.

We prove sufficiency for $r=2$, the generalization to arbitrary $r \in \mathbb{Z}_{+}$ will then be clear.

Since we are dealing with series with positive coefficients we need only consider

$$
\sum_{m_{1}, m_{2}} \hat{\varrho}\left(\Lambda_{1}^{m_{1}} \Lambda_{2}^{m_{2}}\right) \frac{t_{1}^{m_{1}}}{\left(2 m_{1}\right) !} \frac{t_{2}^{m_{2}}}{\left(2 m_{2}\right) !}=\sum_{m} \sum_{m_{1}+m_{2}=m} \hat{\varrho}\left(\Lambda_{1}^{m_{1}} \Lambda_{2}^{m_{2}}\right) \frac{t_{1}^{m_{1}}}{\left(2 m_{1}\right) !} \frac{t_{2}^{m_{2}}}{\left(2 m_{2}\right) !}
$$

where $t_{1}, t_{2}>0$.

For $m_{1}+m_{2}=m$ we have

$$
\begin{aligned}
\frac{t_{1}^{m_{1}}}{\left(2 m_{1}\right) !} \frac{t_{2}^{m_{2}}}{\left(2 m_{2}\right) !} & =\frac{\left(\sqrt{t_{1}}\right)^{2 m_{1}}}{\left(2 m_{1}\right) !} \frac{\left(\sqrt{t_{2}}\right)^{2 m_{2}}}{\left(2 m_{2}\right) !} \leqq \frac{\left(\sqrt{t_{1}}+\sqrt{t_{2}}\right)^{2 m}}{(2 m) !} \\
& =\frac{\left[\left(\sqrt{t_{1}}+\sqrt{t_{2}}\right)^{2}\right]^{m}}{(2 m) !}
\end{aligned}
$$


We also note that

$$
\bigcup_{m_{1}+m_{2}=m} \Lambda_{1}^{m_{1}} \Lambda_{2}^{m_{2}} \subset\left(\Lambda_{1} \cup \Lambda_{2}\right)^{m}
$$

and, hence, that

$$
\sum_{m_{1}+m_{2}=m} \hat{\varrho}\left(\Lambda_{1}^{m_{1}} \Lambda_{2}^{m_{2}}\right) \leqq \hat{\varrho}\left(\Lambda_{1} \cup \Lambda_{2}\right)^{m} .
$$

Let $\Lambda_{1} \cup \Lambda_{2}=\Lambda \in \mathscr{R}$. Then, by assumption,

$$
\sum_{m=0}^{\infty} \hat{\varrho}\left(\Lambda^{m}\right) \frac{z^{m}}{(2 m) !}
$$

has a non-zero radius of convergence. Call it $R_{\Lambda}$. Clearly if $\sqrt{t_{1}}<\frac{1}{2} \sqrt{R_{A}}$ and $\sqrt{t_{2}}<\frac{1}{2} \sqrt{R_{A}}$ the original series converges.

Thus C.C. implies the convergence, in some region about the origin, of the power series

$$
\sum_{m_{1}, \ldots, m_{r}} \hat{\varrho}\left(\Lambda_{1}^{m_{1}} \ldots \Lambda_{r}^{m_{r}}\right) \frac{z_{1}^{m_{1}}}{\left(2 m_{1}\right) !} \cdots \frac{z_{r}^{m_{r}}}{\left(2 m_{r}\right) !} .
$$

Now, for $\Lambda_{1}, \ldots, \Lambda_{r}$ pairwise disjoint,

$$
\begin{aligned}
\hat{\varrho}\left(\Lambda_{1}^{m_{1}} \ldots \Lambda_{r}^{m_{r}}\right) & =\int_{X} \hat{N}_{\Lambda_{1}^{m_{1}} \ldots \Lambda_{r}^{m_{r}}} d \mu=\int_{X}\left(\hat{N}_{\Lambda_{1}}\right)^{m_{1}} \ldots\left(\hat{N}_{\Lambda_{r}}\right)^{m_{r}} d \mu \\
& =\sum_{n_{1}, \ldots, n_{r}}\left(n_{1}\right)^{m_{1}} \ldots\left(n_{r}\right)^{m_{r}} \mu\left(C_{n_{1}, \ldots, n_{r}}^{\Lambda_{1}, \ldots, \Lambda_{r}} .\right.
\end{aligned}
$$

Plugging this formula into the preceding one and [assuming $\left(z_{1}, \ldots, z_{r}\right)$ is in the region of absolute convergence] interchanging summations we obtain

$$
\begin{aligned}
\sum_{n_{1}, \ldots, n_{r}} \sum_{m_{1}, \ldots, m_{r}} \mu\left(C_{n_{1}, \ldots, n_{r}}^{\Lambda_{1}, \ldots, A_{r}}\right) \frac{\left(n_{1} z_{1}\right)^{m_{1}}}{\left(2 m_{1}\right) !} \cdots \frac{\left(n_{r} z_{r}\right)^{m_{r}}}{\left(2 m_{r}\right) !} \\
=\sum_{n_{1}, \ldots, n_{r}} \mu\left(C_{n_{1}, \ldots, n_{r}}^{\Lambda_{1}, \ldots, \Lambda_{r}}\right) \cosh \sqrt{n_{1} z_{1}} \ldots \cosh \sqrt{n_{r} z_{r}} .
\end{aligned}
$$

Proposition 4.3. Let the series

$$
\sum_{n_{1}, \ldots, n_{r}} a_{n_{1}, \ldots, n_{r}} \cosh \sqrt{n_{1} z_{1}} \ldots \cosh \sqrt{n_{r} z_{r}}
$$

be convergent in the region in $\mathbb{C}^{r}$

$$
\left|z_{i}\right|<R i=1, \ldots, r
$$

and suppose the series

$$
\sum_{n_{1}, \ldots, n_{r}}\left|a_{n_{1} \ldots n_{r}}\right| \cosh \sqrt{n_{1} t_{1}} \ldots \cosh \sqrt{n_{r} t_{r}}
$$


converges for

If

$$
t_{i} \in[0, R) \quad i=1, \ldots, r .
$$

$$
\sum_{n_{1}, \ldots, n_{r}} a_{n_{1} \ldots n_{r}} \cosh \sqrt{n_{1} z_{1}} \ldots \cosh \sqrt{n_{r} z_{r}} \equiv 0
$$

in this region, then

$$
a_{n_{1} \ldots n_{r}}=0 \forall\left(n_{1}, \ldots, n_{r}\right) \in \mathbb{Z}_{+}^{r} .
$$

Proof. We first show that it is sufficient to prove the theorem for one complex variable. To this end we assume the theorem holds for $r=1$ and suppose

$$
\sum_{n_{1}, \ldots, n_{r}} a_{n_{1} \ldots n_{r}} \cosh \sqrt{n_{1} z_{1}} \ldots \cosh \sqrt{n_{r} z_{r}}
$$

is identically zero for $\left|z_{i}\right|<R$. Fix $z_{1}, \ldots, z_{r-1}$ in this region and consider the series in $z_{r}$ :

where

$$
\sum_{n_{r}} b_{n_{r}} \cosh \sqrt{n_{r} z_{r}}
$$

$$
b_{n_{r}}:=\sum_{n_{1}, \ldots, n_{r-1}} a_{n_{1} \ldots n_{r}} \cosh \sqrt{n_{1} z_{1}} \ldots \cosh \sqrt{n_{r-1} z_{r-1}} .
$$

Because of absolute convergence we can perform the sums in an arbitrary manner. Hence

$$
\sum_{n_{r}} b_{n_{r}} \cosh \sqrt{n_{r} z_{r}}
$$

is identically zero for $\left|z_{r}\right|<R$. Moreover

$$
\sum_{n_{r}}\left|b_{n_{r}}\right| \cosh \sqrt{n_{r} t_{r}}
$$

is convergent for $t_{r} \in[0, R)$. This follows from

$$
\left|b_{n_{r}}\right| \leqq \sum_{n_{1}, \ldots, n_{r-1}}\left|a_{n_{1} \ldots n_{r}}\right| \cosh \sqrt{n_{1}\left|z_{1}\right|} \ldots \cosh \sqrt{n_{r-1}\left|z_{r-1}\right|}
$$

where we have used the fact that

$$
|\cosh z| \leqq \cosh (\operatorname{Re} z) \leqq \cosh |z| .
$$

Since we have assumed the statement is true for $r=1$ we have

That is:

$$
b_{n_{r}}=0 \text { all } n_{r} .
$$

$$
\sum_{n_{1} \ldots n_{r-1}} a_{n_{1} \ldots n_{r}} \cosh \sqrt{n_{1} z_{1}} \ldots \cosh \sqrt{n_{r-1} z_{r-1}}=0 \forall n_{r} \in \mathbb{Z}_{+} .
$$

This clearly holds for all $\left|z_{i}\right|<R \quad i=1, \ldots, r-1$. We continue arguing in the same way to obtain $a_{n_{1} \ldots n_{r}}=0\left(n_{1}, \ldots, n_{r}\right) \in \mathbb{Z}_{+}^{r}$. 
We now proceed to prove the proposition for a single complex variable. It is convenient to change variables to

$$
\zeta=-i \sqrt{z}
$$

and consider the function defined for $|\zeta|<\sqrt{R}$ by

$$
g(\zeta)=\sum_{n=0}^{\infty} a_{n} \cosh n^{\frac{1}{2}} i \zeta=\sum_{n=0}^{\infty} a_{n} \cos n^{\frac{1}{2}} \zeta .
$$

Then $g(\zeta)$ is identically zero for $|\zeta|<\sqrt{R}$ and $\sum_{n=0}^{\infty}\left|a_{n}\right||\cos \sqrt{n} t|$ converges for all $t \in \mathbb{R}$ since

$$
\sum_{n=0}^{\infty}\left|a_{n}\right|<\infty .
$$

[This follows from the fact that $\cosh \sqrt{n} t \geqq 1$.]

Since

we see that

$$
|\cos \sqrt{n} \zeta| \leqq \cosh (\sqrt{n} \mathfrak{T} m \zeta)
$$

$$
\sum_{n=0}^{\infty} a_{n} \cos \sqrt{n} \zeta
$$

converges absolutely in the strip in the complex $\zeta$ plane

$$
|\mathfrak{I} m \zeta|<\sqrt{R} \text {. }
$$

Moreover it is easy to see that it converges uniformly on compact sets in this region. Hence $g(\zeta)$ is defined as an analytic function in the strip $|\mathfrak{T} m \zeta|<\sqrt{R}$ and coincides with the analytic function "Zero" in the region $|\zeta|<\sqrt{R}$. Thus, by the uniqueness of the analytic continuation of an analytic function, $g(\zeta)=0$ for all $\zeta$ with $|\mathfrak{T} m \zeta|<\sqrt{R}$.

Consider $g(\zeta)$ on the real axis,

Since

$$
g(t)=\sum_{n=0}^{\infty} a_{n} \cos \sqrt{n} t=0 \quad \text { all } \quad t \in \mathbb{R} .
$$

$$
\sum_{n=0}^{\infty}\left|a_{n}\right|<\infty
$$

the series for $g(t)$ converges uniformly on $\mathbb{R}$. Hence $g(t)$ is an almost periodic function with "spectral points" $(\sqrt{n})_{n=0}^{\infty}$. Such functions have unique Fourier like expansions of the type above. Since $g$ is the Zero function the coefficients of its expansion must vanish. Hence

$$
a_{n}=0 \forall n \in \mathbb{N} .
$$

Proposition 4.4. Let the sequence of measures $\left(\hat{\varrho}_{n}\right)_{n=1}^{\infty}$ satisfy C.C. Then $\mu$ is uniquely associated with $\left(\hat{\varrho}_{n}\right)_{n=1}^{\infty}$. 
Proof. Suppose there are two measures $\mu_{1}$ and $\mu_{2}$ associated with $\left(\varrho_{n}\right)_{n=1}^{\infty}$. Then we have the equality of the two series

$$
\sum_{n_{1}, \ldots, n_{r}} \mu_{i}\left(C_{n_{1}, \ldots, n_{r}}^{A_{1}, \ldots, A_{r}}\right) \cosh \sqrt{n_{1} z_{1}} \ldots \cosh \sqrt{n_{r} z_{r}} \quad i=1,2
$$

for any cylinder set

and

$$
C_{n_{1}, \ldots, n_{r}}^{\Lambda_{1}, \ldots, \Lambda_{r}}
$$

$$
\left|z_{i}\right|<R
$$

[R of course depends on the pairwise disjoint sets $\left.\Lambda_{1}, \ldots, \Lambda_{r}\right]$.

C.C. clearly implies the convergence of

$$
\sum_{n_{1}, \ldots, n_{r}} \mu_{i}\left(C_{n_{1}, \ldots, n_{r}}^{\Lambda_{1}, \ldots, \Lambda_{r}}\right) \cosh \sqrt{n_{1} t_{1}} \ldots \cosh \sqrt{n_{r} t_{r}}, \quad i=1,2
$$

in this region. Hence in subtracting the two series we can subtract term by term. Letting

we have

$$
a_{n_{1} \ldots n_{r}}=\mu_{1}\left(C_{n_{1}, \ldots, n_{r}}^{\Lambda_{1}, \ldots, A_{r}}\right)-\mu_{2}\left(C_{n_{1}, \ldots, n_{r}}^{\Lambda_{1}, \ldots, A_{r}}\right)
$$

$$
\sum_{n_{1}, \ldots, n_{r}} a_{n_{1} \ldots n_{r}} \cosh \sqrt{n_{1} z_{1}} \ldots \cosh \sqrt{n_{r} z_{r}}=0
$$

while clearly

$$
\sum_{n_{1}, \ldots, n_{r}}\left|a_{n_{1} \ldots n_{r}}\right| \cosh \sqrt{n_{1} t_{1}} \ldots \cosh \sqrt{n_{r} t_{r}}<\infty \quad \text { for } t_{i} \in[0, R) .
$$

We now simply apply proposition (4.3) to conclude that the coefficients vanish identically. Thus $\mu_{1}=\mu_{2}$ on the cylinder sets and hence on $\Sigma[\mathbb{C}]$.

\section{§ 5. The Relationship between $\mu$ and $A_{\Im}$}

In this section we strengthen our assumption on $\Omega$ to analyticity for every operator

$$
A_{\varphi_{\Lambda}}, \Lambda \in \mathscr{R} .
$$

This allows us to establish essential self-adjointness of these operators on $\mathscr{D}$ and obtain an expression for $\mu$ on the cylinder sets in terms of the associated spectral measures.

The basic tools which will be used in obtaining these results are theorems concerning the classical moment problems. These and the relevant definitions can be found in Appendix 2.

Our first task is to establish further properties of the $A_{\varphi_{\Lambda}}$. To this end we recall certain types of vectors in a Hilbert space.

Definitions. Let $A$ be an operator in a Hilbert space $\mathfrak{h}$ and $\Psi \in \mathfrak{h}$ for which $A^{n} \Psi$ is defined for every $n$. 
i) $\Psi$ is analytic for $A$ if the power series

$$
\sum_{n=0}^{\infty} \frac{\left\|A^{n} \Psi\right\|}{n !} z^{n}
$$

has a non-zero radius of convergence.

ii) $\Psi$ is Stieltjes for $A$ if

$$
\sum_{n=1}^{\infty}\left\|A^{n} \Psi\right\|^{-\frac{1}{2 n}}=\infty .
$$

We state the well known result:

Proposition 5.1. $\Psi$ analytic for $A \Rightarrow \Psi$ semi-analytic for $A \Rightarrow \Psi$ Stieltjes for $A$.

We now make the assumption that $\Omega$ is an analytic vector for $A_{\varphi_{\Lambda}}, \Lambda \in \mathscr{R}$.

Proposition 5.2. $\Omega$ analytic for $A_{\varphi_{\Lambda}}$ implies $\Psi_{\varphi} \in \mathscr{D}$ is semi-analytic for $A_{\varphi_{A}}$.

Proof. Since $\Psi_{\varphi}$ is a finite linear combination of vectors of the form $\Psi_{\varphi A_{1} \ldots A_{r}}$ the triangle inequality shows that it suffices to prove the proposition for vectors of this form. Recall

We have

$$
\Psi_{\varphi_{\Lambda_{1} \ldots \Lambda_{r}}}=A_{\varphi_{\Lambda_{1} \ldots \Lambda_{r}}} \Omega
$$

$$
\begin{aligned}
\left\|A_{\varphi_{\Lambda}}^{n} A_{\varphi_{\Lambda_{1}} \ldots \Lambda_{r}} \Omega\right\| & =(\underbrace{A_{\varphi_{1} \otimes \ldots \otimes \varphi_{A}}}_{2 n \text { times }} A_{\varphi_{\Lambda_{1} \ldots \Lambda_{r}} \otimes \varphi_{\Lambda_{1} \ldots \Lambda_{r}}} \Omega, \Omega)^{\frac{1}{2}} \\
& =\hat{\varrho}\left(\left(\varphi_{\Lambda}\right)^{2 n} \otimes\left(\varphi_{\Lambda_{1} \ldots \Lambda_{r}}\right)^{2}\right)^{\frac{1}{2}} \\
& =\hat{\varrho}\left(\Lambda^{2 n}\left(\Lambda_{1} \ldots \Lambda_{r}\right)^{2}\right)^{\frac{1}{2}} \\
& =\left[\int \hat{N}_{\Lambda}^{2 n} \hat{N}_{\Lambda_{1} \ldots \Lambda_{r}}{ }^{2} d \mu\right]^{\frac{1}{2}} .
\end{aligned}
$$

Since $\hat{N}_{A}^{2 n}$ and $\hat{N}_{\Lambda_{1} \ldots \Lambda_{r}^{2}}$ are elements of $\mathscr{L}_{2}(X, \Sigma[\mathbb{C}], \mu)$ we may apply Cauchy-Schwarz to obtain

\section{Letting}

$$
\left\|A_{\varphi_{\Lambda}}^{n} A_{\varphi_{\Lambda_{1} \ldots A_{r}}} \Omega\right\| \leqq\left\|\hat{N}_{\Lambda_{1} \ldots \Lambda_{r}}^{2}\right\|^{\frac{1}{2}}\left\|\hat{N}_{\Lambda}^{2 n}\right\|^{\frac{1}{2}} .
$$

we write

$$
X_{\Lambda}=\left\{\xi \in X: \hat{N}_{\Lambda}(\xi) \neq 0\right\}=\left\{\xi \in X: \hat{N}_{\Lambda}(\xi) \geqq 1\right\}
$$

to obtain

$$
\left\|\hat{N}_{\Lambda}^{2 n}\right\|=\left[\mu\left(X_{\Lambda}\right) \int_{X_{\Lambda}} \hat{N}_{\Lambda}^{4 n} \frac{d \mu}{\mu\left(X_{\Lambda}\right)}\right]^{\frac{1}{2}}
$$

$$
\begin{aligned}
\left\|\hat{N}_{\Lambda}^{2 n}\right\|^{\frac{1}{2}} & =\hat{\varrho}\left(\Lambda^{4 n}\right)^{\frac{1}{4}} \leqq \mu\left(X_{\Lambda}\right)^{\frac{1}{4}}\left[\int_{X_{\Lambda}} \hat{N}_{\Lambda}^{4 n} \frac{d \mu}{\mu\left(X_{\Lambda}\right)}\right]^{\frac{1}{2}}=\frac{1}{\mu\left(X_{\Lambda}\right)^{\frac{1}{4}}} \hat{\varrho}\left(\Lambda^{4 n}\right)^{\frac{1}{2}} \\
& =\frac{1}{\mu\left(X_{\Lambda}\right)^{\frac{1}{4}}}\left\|A_{\varphi_{\Lambda}}^{2 n} \Omega\right\| .
\end{aligned}
$$


Thus

$$
\left\|A_{\varphi_{\Lambda}}^{n} A_{\varphi_{\Lambda_{1}} \ldots \Lambda_{r}} \Omega\right\| \leqq \text { const. }\left\|A_{\varphi_{\Lambda}}^{2 n} \Omega\right\| .
$$

Since $\Omega$ is analytic the series

$$
\sum_{n=0}^{\infty} \frac{\left\|A_{\varphi_{\Lambda}}^{2 n} \Omega\right\|}{(2 n) !} z^{2 n}
$$

has a non-zero radius of convergence and hence so does the series

$$
\sum_{n=0}^{\infty} \frac{\left\|A_{\varphi_{\Lambda}}^{n} A_{\varphi_{\Lambda_{1} \ldots A_{r}}} \Omega\right\|}{(2 n) !} z^{n} .
$$

Proposition (3.2), (5.2) and a theorem due to Masson and McClary immediately yield

Proposition 5.3. $A_{\varphi_{\Lambda}}, \Lambda \in \mathscr{R}$, is essentially self-adjoint on $\mathscr{D}$.

Proof. The theorem due to Masson and McClary (Ref. [3]) states: Let $A$ be a symmetric operator which is bounded below and has a domain which contains a dense set of Stieltjes vectors. Then $A$ is essentially self-adjoint.

Since semi-analytic vectors are Stieltjes vectors, $\mathscr{D}$ is dense in $\mathscr{H}$, and $A_{\varphi_{A}}$ is positive on $\mathscr{D}$ the result is a direct application of MassonMcClary.

From this point on we let $A_{\varphi_{\Lambda}}$ denote the self-adjoint closure of the operator originally defined on $\mathscr{D}$.

Proposition 5.4. The spectrum of $A_{\varphi_{A}}$ is a subset of the positive integers.

Proof. Since $\operatorname{spec}\left(A_{\varphi_{A}}\right) \subset \mathbb{R}$ we only need to show that if $\lambda \in \mathbb{R}$ and $\lambda \notin \mathbb{Z}_{+}$the operator

$$
A_{\varphi_{\Lambda}}-\lambda
$$

is bounded away from zero. This implies it has a bounded inverse and hence

$$
\lambda \notin \operatorname{spec}\left(A_{\varphi_{A}}\right) .
$$

For $\varphi \in \mathbb{S}$ consider

$$
\begin{aligned}
\left\|\left(A_{\varphi_{\Lambda}}-\lambda\right) \Psi_{\varphi}\right\|^{2} & =\left(\left(A_{\varphi_{\Lambda}}-\lambda\right)^{2} \Psi_{\varphi}, \Psi_{\varphi}\right) \\
& =\int_{X}\left(\hat{N}_{\Lambda}-\lambda\right)^{2}\left|\hat{\eta}_{\varphi}\right|^{2} d \mu .
\end{aligned}
$$

Since $\hat{N}_{A}$ is a non-negative integer valued function and we have assumed $\lambda$ is not a positive integer there exists $k>0$ such that

$$
\left(\hat{N}_{\Lambda}(\xi)-\lambda\right)^{2} \geqq k^{2} \quad \forall \xi \in X .
$$


Thus

i.e.

$$
\left\|\left(A_{\varphi_{\Lambda}}-\lambda\right) \Psi_{\varphi}\right\|^{2} \geqq k^{2} \int_{X}\left|\hat{\eta}_{\varphi}\right|^{2} d \mu
$$

$$
\left\|\left(A_{\varphi_{\Lambda}}-\lambda\right) \Psi_{\varphi}\right\| \geqq k\left\|\Psi_{\varphi}\right\|
$$

for every $\Psi_{\varphi} \in \mathscr{D}$. Since $A_{\varphi_{A}}$ is essentially self-adjoint on $\mathscr{D}$ this inequality extends to every $\Psi \in \operatorname{Dom}\left(A_{\varphi_{A}}\right)$, showing that $A_{\varphi_{\Lambda}}-\lambda$ has a bounded inverse.

We state without proof the following easy result.

Lemma. Let $A$ be a positive operator defined on a Hilbert space $\mathfrak{h}$. Let $\Psi \in \mathfrak{h}$ be a Stieltjes vector for $A$. Let $S_{k} \equiv\left(A^{k} \Psi, \Psi\right) k=1,2, \ldots$ Then

$$
\sum_{k=1}^{\infty} S_{k}^{-\frac{1}{2 k}}=\infty .
$$

Theorem 5.5. Let $\mathfrak{h}$ be $a$ Hilbert space and $A$ and $B$ two positive unbounded symmetric operators defined on a common, dense, invariant domain, $\mathscr{D}$, of vectors in $\mathfrak{h}$ which are Stieltjes for both $A$ and $B$. Suppose that $A$ and $B$ commute on $\mathscr{D}$. Then the spectral measures associated with $A$ and $B$ commute.

Proof. By the Massen-McClary Theorem A and B are essentially self-adjoint on $\mathscr{D}$. Hence they are each associated with a spectral measure. We denote by $E$ and $F$ the spectral measures associated with $\mathrm{A}$ and $\mathrm{B}$ respectively. We shall show that if $M$ and $N$ are Borel sets in $\mathbb{R}$ such that $E(M) \neq 0$ and $F(N) \neq 0$ then for $\Psi \in \mathscr{D}$

$$
(E(M) F(N) \Psi, \Psi)=(F(N) E(M) \Psi, \Psi) .
$$

This is obviously necessary and sufficient to show that $E(M)$ commutes with $F(N)$. (5.1) is obviously equivalent to

$$
(E(M) \Psi, F(N) \Psi)=(F(N) \Psi, E(M) \Psi) .
$$

For $\Psi \in \mathscr{D}$ with $\|\Psi\|=1$ let $\sigma_{E}^{\Psi}$ be the measure on $\mathbb{R}$ defined by

$$
\sigma_{E}^{\Psi}(\cdot)=(E(\cdot) \Psi, \Psi)
$$

We consider $\mathscr{L}_{2}\left(\sigma_{E}^{\Psi}\right)$. Since $\mathscr{D}$ is invariant under $A \Psi \in C^{\infty}(A)$. Let

$$
S_{k}=\int_{0}^{\infty} \lambda^{k} d \sigma_{E}^{\Psi}=\left(A^{k} \Psi, \Psi\right)
$$

Then $\left(S_{k}\right)_{k=0}^{\infty}$ is a sequence of real numbers with $S_{0}=1$. By the preceeding lemma this sequence satisfies

$$
\sum_{k=1}^{\infty}\left|S_{k}\right|^{-\frac{1}{2 k}}=\infty .
$$


Thus by Theorem (A.2-A), Proposition (A.2-1), and Theorem (A.2-B) the polynomials are dense in $\mathscr{L}_{2}\left(\sigma_{E}^{\Psi}\right)$.

Let $\chi_{M}$ be the characteristic function of $M$. Then $\chi_{M} \in \mathscr{L}_{2}\left(\sigma_{E}^{\Psi}\right)$. Let $\left(P_{n}\right)_{n=0}^{\infty}$ be a sequence of polynomials which converges to $\chi_{M}$ in $\mathscr{L}_{2}\left(\sigma_{E}^{\Psi}\right)$. Since $\chi_{M}$ is real valued the polynomials can be chosen to be real. We have

That is

$$
\lim _{n \rightarrow \infty} \int\left|P_{n}-\chi_{M}\right|^{2} d \sigma_{E}^{\Psi}=0
$$

$$
\lim _{n \rightarrow \infty}\left\|\left(P_{n}(A)-E(M)\right) \Psi\right\|=0 .
$$

Thus $P_{n}(A) \Psi$ converges strongly to $E(M) \Psi$. By similar arguments concerning $\mathscr{L}_{2}\left(\sigma_{F}^{\Psi}\right)$ we obtain a sequence of polynomials $\left(Q_{n}\right)_{n=0}^{\infty}$ satisfying $Q_{n}(B) \Psi$ converges strongly to $F(N) \Psi$. Thus

$$
\begin{aligned}
(E(M) \Psi, F(N) \Psi) & =\lim _{m \rightarrow \infty} \lim _{n \rightarrow \infty}\left(P_{n}(A) \Psi, Q_{m}(B) \Psi\right) \\
& =\lim _{m \rightarrow \infty} \lim _{n \rightarrow \infty}\left(Q_{m}(B) P_{n}(A) \Psi, \Psi\right) \\
& =\lim _{m \rightarrow \infty} \lim _{n \rightarrow \infty}\left(P_{n}(A) Q_{m}(B) \Psi, \Psi\right) \\
& =\lim _{m \rightarrow \infty} \lim _{n \rightarrow \infty}\left(Q_{m}(B) \Psi, P_{n}(A) \Psi\right) \\
& =(F(N) \Psi, E(M) \Psi) .
\end{aligned}
$$

We are now in a position to obtain the desired expression for $\mu$ on the cylinder sets.

Let $\mathfrak{F}$ be any function defined on the real line and consider

$$
\mathfrak{F} \circ \hat{N}_{\Lambda}, \quad \Lambda \in \mathscr{R}
$$

where "o" denotes composition:

$$
\left(\mathfrak{F} \circ \hat{N}_{\Lambda}\right)(\xi):=\mathfrak{F}\left(\hat{N}_{\Lambda}(\xi)\right)
$$

Clearly $\mathfrak{F} \circ \hat{N}_{A}$ is a function defined on $X$ and

$$
\int\left|\mathfrak{F} \circ \hat{N}_{\Lambda}\right|^{2} d \mu=\sum_{m=0}^{\infty} \mu\left(C_{\Lambda}^{m}\right)|\mathfrak{F}(m)|^{2} .
$$

Let $\ell_{2}(\mu, \Lambda)$ denote the Hilbert space of sequences of complex numbers $\left(\alpha_{m}\right)_{m=0}^{\infty}$ satisfying

Thus

$$
\sum_{m=0}^{\infty} \mu\left(C_{\Lambda}^{m}\right)\left|\alpha_{m}\right|^{2}<\infty
$$

if and only if

$$
\mathfrak{F} \circ \hat{N}_{\Lambda} \in \mathscr{L}_{2}(X, \Sigma[\mathbb{C}], \mu)
$$

$$
(\mathfrak{F}(m))_{m=0}^{\infty} \in \ell_{2}(\mu, \Lambda) \text {. }
$$

Let $\sigma_{\Lambda}^{\Omega}$ denote the measure defined by the cyclic vector $\Omega$ and the spectral measure associated with $A_{\varphi_{\Lambda}}$. 
Proposition 5.6. The polynomials are dense in $\mathscr{L}_{2}\left(\sigma_{\Lambda}^{\Omega}\right), \Lambda \in \mathscr{R}$.

Proof. By assumption $\Omega$ is analytic for $A_{\varphi_{\Lambda}}, \Lambda \in \mathscr{R}$. Hence by Proposition (5.1) it is Stieltjes. We now argue exactly as in the proof of Theorem (5.5).

Proposition 5.7. $\mathscr{L}_{2}\left(\sigma_{A}^{\Omega}\right)$ is isomorphic to a subspace of $\ell_{2}(\mu, \Lambda)$.

Proof. By the previous proposition it suffices to show that the polynomials are mapped isometrically into $\ell_{2}(\mu, \Lambda)$ since an isometric mapping of a dense linear manifold of one Hilbert space into another clearly extends to a mapping of the whole Hilbert space into the other.

Consider

$$
\begin{aligned}
\int \lambda^{n} d \sigma_{\Lambda}^{\Omega} & =\left(A_{\varphi_{A}}^{n} \Omega, \Omega\right) \\
& =\left(A_{\varphi_{\Lambda^{n}}} \Omega, \Omega\right) \\
& =\hat{\varrho}\left(\varphi_{\Lambda^{n}}\right) \\
& =\int_{X} \hat{N}_{\Lambda}^{n} d \mu \\
& =\sum_{m=0}^{\infty} \mu\left(C_{m}^{A}\right) m^{n} .
\end{aligned}
$$

Let $\pi$ be an arbitrary polynomial in $\mathscr{L}_{2}\left(\sigma_{A}^{\Omega}\right)$. The above equality obviously generalizes to

$$
\int|\pi(\lambda)|^{2} d \sigma_{\Lambda}^{\Omega}=\sum_{m=0}^{\infty} \mu\left(C_{m}^{\Lambda}\right)|\pi(m)|^{2} .
$$

This formula explicitly exhibits both the mapping of $\mathscr{L}_{2}\left(\sigma_{\Lambda}^{\Omega}\right)$ into $\ell_{2}(\mu, \Lambda)$ and its isometric nature.

For $m \in \mathbb{Z}_{+}$let $p_{m}:=\left(E_{\Lambda}(m) \Omega, \Omega\right)$. Where $E_{\Lambda}$ denotes the spectral measure of $A_{\varphi_{A}}$. We know from spectral theory that

i) $\sum_{m=0}^{\infty} p_{m}=1$ and

ii) $p_{m} \neq 0$ if and only if $m \in \operatorname{spec}\left(A_{\varphi_{A}}\right)$.

The next proposition exhibits the relationship between $\operatorname{spec}\left(A_{\varphi_{A}}\right)$ and $\left(C_{m}^{\Lambda}\right)_{m=0}^{\infty}$.

Proposition 5.8. $m \in \operatorname{spec}\left(A_{\varphi_{A}}\right)$ if and only if $\mu\left(C_{m}^{A}\right) \neq 0$.

Proof. We again consider the [determinate] moment problem

$$
\begin{aligned}
S_{k}=\left(A_{\varphi_{\Lambda}}^{k} \Omega, \Omega\right)=\int \lambda^{k} d \sigma_{\Lambda}^{\Omega} & =\sum_{m} m^{k}\left(E_{\Lambda}(m) \Omega, \Omega\right) \\
& =\sum_{m} m^{k} p_{m} .
\end{aligned}
$$


Thus we have the two expression for $S_{k}$

and

$$
S_{k}=\sum_{m=0}^{\infty} m^{k} p_{m}
$$

$$
S_{k}=\sum_{m=0}^{\infty} m^{k} \mu\left(C_{m}^{\Lambda}\right) .
$$

Since these sums are merely special cases of integrals we conclude, since the moment problem is determinate,

$$
\mu\left(C_{m}^{\Lambda}\right)=p_{m} .
$$

This equality plus ii) above yields the proposition.

The equality

$$
\mu\left(C_{m}^{\Lambda}\right)=p_{m}
$$

establishes the complete one to one correspondence between $\mathscr{L}_{2}\left(\sigma_{A}^{\Omega}\right)$ and $\ell_{2}(\mu, \Lambda)$. Since

$$
\ell_{2}(\mu, \Lambda) \subset \mathscr{L}_{2}(X, \Sigma[\mathbb{C}], \mu)
$$

we may conclude that if

$$
f \in \mathscr{L}_{2}\left(\sigma_{\Lambda}^{\Omega}\right)
$$

and $\left(\pi_{n}\right)_{n=0}^{\infty}$ a sequence of polynomials that converges strongly to $f$ then $\left(\pi_{n} \circ \hat{N}_{\Lambda}\right)$ converges strongly to $f \circ \hat{N}_{\Lambda}$ in $\mathscr{L}_{2}(X, \Sigma[\mathbb{C}], \mu)$.

We have now assembled the machinery necessary to recover $\mu$ on all cylinder sets. This is our final result.

Theorem 5.9. Let

$$
\Lambda_{1}, \Lambda_{2}, \ldots, \Lambda_{r} \text { be disjoint sets in } \mathscr{R} \text { and let } E_{i}
$$

denote the spectral measure of $A_{\varphi_{\Lambda_{i}}}, i=1, \ldots, r$. Then, for positive integers $m_{1}, \ldots, m_{r}$,

$$
\mu\left(C_{m_{1}, \ldots, m_{r}}^{\Lambda_{1}, \ldots, A_{r}}\right)=\left(E_{1}\left(m_{1}\right) \ldots E_{r}\left(m_{r}\right) \Omega, \Omega\right)
$$

Proof. Choose sequences of polynomials $\left(\pi_{k}^{(i)}\right), i=2, \ldots, r$ so that $\left(\pi_{k}^{(i)}\right)$ converges strongly to $\chi_{\left\{m_{1}\right\}}$ on $\mathscr{L}_{2}\left(\sigma_{\Lambda_{i}}^{\Omega}\right)$. Then $\left(\pi_{k}^{(i)} \circ \hat{N}_{\Lambda_{1}}\right)$ converges strongly to $\chi_{\left\{m_{1}\right\}} \circ \hat{N}_{\Lambda_{i}}$ in $\mathscr{L}_{2}(X, \Sigma[\mathcal{C}], \mu)$. Moreover the operator polynomials $\pi_{k}^{(i)}\left(A_{\varphi_{\Lambda_{i}}}\right)$ satisfy

Consider

$$
s \cdot \lim _{k \rightarrow \infty} \pi_{k}^{(i)}\left(A_{\varphi_{\Lambda_{i}}}\right) \Omega=E_{i}\left(m_{i}\right) \Omega, \quad i=2, \ldots, r .
$$

$$
S_{n}:=\lim _{k_{2} \rightarrow \infty} \lim _{k_{3} \rightarrow \infty} \lim _{k_{r} \rightarrow \infty}\left(A_{\varphi_{\Lambda_{1}}}^{n} \pi_{k_{2}}^{(2)}\left(A_{\varphi_{\Lambda_{2}}}\right) \ldots \pi_{k_{r}}^{(r)}\left(A_{\varphi_{\Lambda_{r}}}\right) \Omega, \Omega\right) .
$$


The above formula and Theorem (5.5) yield

Noting that

$$
\begin{aligned}
S_{n} & =\left(A_{\varphi_{\Lambda_{1}}}^{n} E_{2}\left(m_{2}\right) \ldots E_{r}\left(m_{r}\right) \Omega, \Omega\right) \\
& =\sum_{m_{1}}\left(m_{1}\right)^{n}\left(E_{1}\left(m_{1}\right) E_{2}\left(m_{2}\right) \ldots E_{r}\left(m_{r}\right) \Omega, \Omega\right) .
\end{aligned}
$$

$$
\begin{aligned}
\left(A_{\varphi_{\Lambda_{1}}}^{n} \pi_{k_{2}}^{(2)}\left(A_{\varphi_{\Lambda_{2}}}\right)\right. & \left.\ldots \pi_{k_{r}}^{(r)}\left(A_{\varphi_{\Lambda_{r}}}\right) \Omega, \Omega\right) \\
& =\int_{X}\left(\hat{N}_{\Lambda_{1}}\right)^{n} \pi_{k_{2}}^{(2)} \circ \hat{N}_{\Lambda_{2}} \ldots \pi_{k_{r}}^{(r)} \circ \hat{N}_{\Lambda_{r}} d \mu
\end{aligned}
$$

we obtain the alternative expression for $S_{n}$ :

$$
\begin{aligned}
S_{n} & =\int_{X}\left(\hat{N}_{\Lambda_{1}}\right)^{n} \chi_{\left\{m_{2}\right\}} \circ \hat{N}_{\Lambda_{2}} \ldots \chi_{\left\{m_{r}\right\}} \circ \hat{N}_{A_{r}} d \mu \\
& =\int\left(\hat{N}_{A_{1}}\right)^{n} d \mu \\
& \cdot C_{m_{2}, \ldots, \ldots, m_{r}}^{\Lambda_{2}, \ldots, A_{r}} \\
& =\sum_{m_{1}}\left(m_{1}\right)^{n} \mu\left(C_{m_{1}, m_{2}, \ldots, m_{r}}^{\Lambda_{1}, A_{2}, \ldots, A_{r}} .\right.
\end{aligned}
$$

Since $E_{2}\left(m_{2}\right) \ldots E_{r}\left(m_{r}\right) \Omega$ is clearly analytic for $A_{\varphi_{\Lambda_{1}}}$ we again have

$$
\sum_{n=1}^{\infty}\left|S_{n}\right|^{-\frac{1}{2 n}}=\infty \text {. }
$$

Thus Carleman's result again tells us that the moment problem associated with the sequence $\left(S_{n}\right)$ is determinate and hence we conclude that

$$
\mu\left(C_{m_{1}, \ldots, m_{r}}^{\Lambda_{1}, \ldots, A_{r}}\right)=\left(E_{1}\left(m_{1}\right) \ldots E_{r}\left(m_{r}\right) \Omega, \Omega\right) .
$$

\section{Appendix 1: On the Definition of the Modified Correlation Measures}

We consider correlation functions

$$
\left(\varrho_{n}\right)_{n=1}^{\infty}
$$

where $\varrho_{n}\left(x_{1}, \ldots, x_{n}\right)$ is the probability density for the positions of $n$-distinct particles. To construct the modified correlation functions we remove the condition that the particles be distinct. Thus we allow for the possibility of two or more variables in fact describing the same particle. For $n=2$ we have

$$
\hat{\varrho}_{2}\left(x_{1}, x_{2}\right)=\varrho_{2}\left(x_{1}, x_{2}\right)+\varrho_{1}\left(x_{1}\right) \delta\left(x_{1}-x_{2}\right) .
$$

The second term taking into account the possible identity of particles 1 and 2. 
The generalization of the above expression for $n$ variables is

$$
\hat{\varrho}_{n}\left(x_{1}, x_{2}, \ldots, x_{n}\right)=\sum_{m=0}^{n} \sum_{\mathscr{C} \in \mathscr{P}_{n}^{(m)}} \varrho_{m}(\mathscr{C}) \hat{\delta}_{\mathscr{C}}(1,2, \ldots, n) .
$$

Here $\mathscr{P}_{n}^{(m)}$ is the set of partitions of $n$ integers into $m$ disjoint subsets. $\varrho_{m}(\mathscr{C})$ is the $m$-particle correlation function with arguments

determined by $\mathscr{C}$ as follows:

$$
x_{k_{1}}, \ldots, x_{k_{m}}
$$

$i \neq j \Rightarrow k_{i}$ and $k_{j}$ are in different members of $\mathscr{C}$, and $k_{i}$ is the smallest integer in its subset.

$\hat{\delta}_{\mathscr{C}}(1,2, \ldots, n)$ is a product of delta function factors indicating that the variables indexed by integers in the various subsets that comprise $\mathscr{C}$ are to be identified.

Now let $\Lambda_{1}, \ldots, \Lambda_{r}$ be pair-wise disjoint measurable sets in the phase space of the particles and let $m_{1}, \ldots, m_{r}$ be positive integers satisfying

Define

$$
\sum_{i=1}^{r} m_{i}=n \text {. }
$$

and

$$
\varrho_{n}\left(\Lambda_{\Lambda_{1}}^{m_{1}} \ldots \Lambda_{r}^{m_{r}}\right):=\int_{\Lambda_{1}^{m_{1} \ldots \Lambda_{r}^{m_{r}}}} \ldots \varrho_{n}\left(x_{1}, \ldots, x_{n}\right) d x_{1} \ldots d x_{n}
$$

$$
\hat{\varrho}_{n}\left(\Lambda_{1}^{m_{1}} \ldots \Lambda_{r}^{m_{r}}\right):=\int_{\Lambda_{1}^{m_{1} \ldots} \ldots \Lambda_{r}^{m_{r}}} \hat{\varrho}_{n}\left(x_{1}, \ldots, x_{n}\right) d x_{1} \ldots d x_{n} .
$$

Plugging in the expression for $\hat{\varrho}_{n}$ above and employing a little deltafunction mathematics and combinatoric analysis yields the expression

$$
\varrho_{n}\left(\Lambda_{1}^{m_{1}} \ldots \Lambda_{r}^{m_{r}}\right)=\sum_{k_{1}=0}^{m_{1}} \ldots \sum_{k_{r}=0}^{m_{r}} \prod_{i=1}^{r} \mathscr{S}_{m_{i}}^{\left(k_{i}\right)} \varrho_{\Sigma k_{i}}\left(\Lambda_{1}^{k_{1}} \ldots \Lambda_{r}^{k_{r}}\right) .
$$

[We adopt the convention: $\Lambda^{0} \Lambda^{\prime k} \equiv \Lambda^{\prime k}$.]

Here $\mathscr{S}_{n}^{(m)}$ is the Stirling number of the second kind and is the number of ways of partitioning a set of $n$ elements into $m$ non-empty disjoint subsets.

We now have an expression in terms of correlation measures. We adopt this as the definition of the modified correlation measures on the sets $\Lambda_{1}^{m_{1}} \ldots \Lambda_{r}^{m_{r}}$. Our goal is to obtain an expression for $\hat{\varrho}_{n}$ similar to that for $\varrho_{n}$, i.e., find a function $\hat{N}_{H}$ such that

$$
\hat{\varrho}_{n}(H)=\int_{X} \hat{N}_{H} d \mu .
$$

We substitute into the above expression for $\hat{\varrho}_{n}$ the formula (see Ref. [2])

$$
\left.\varrho_{\Sigma k_{i}}\left(\Lambda_{1}^{k_{1}} \ldots \Lambda_{r}^{k_{r}}\right)=\int_{X} d \mu \prod_{i=1}^{r}\left(\# \xi_{\Lambda_{i}}\right)\left(\# \xi_{\Lambda_{i}}-1\right) \ldots\left(\# \xi_{\Lambda_{i}}-k_{i+1}\right)\right] .
$$


This yields

$$
\begin{aligned}
\hat{\varrho}_{n}\left(\Lambda_{1}^{m_{1}} \ldots \Lambda_{r}^{m_{r}}\right) & \\
& =\int_{X} d \mu\left[\sum_{k_{1}=0}^{m_{1}} \ldots \sum_{k_{r}=0}^{m_{r}} \prod_{i=1}^{r} \mathscr{S}_{m_{k}}^{\left(k_{i}\right)}\left(\# \xi_{\Lambda_{i}}\right)\left(\# \xi_{\Lambda_{i}}-1\right) \ldots\left(\# \xi_{\Lambda_{i}}-k_{i}+1\right)\right] \\
& =\int_{X} d \mu \prod_{i=1}^{r}\left[\sum_{k_{i}=0}^{m_{i}} \mathscr{S}_{m_{i}}^{\left(k_{i}\right)}\left(\# \xi_{A_{i}}\right)\left(\# \xi_{\Lambda_{i}}-1\right) \ldots\left(\# \xi_{\Lambda_{i}}-k_{i}+1\right)\right] .
\end{aligned}
$$

We now use the combinatoric identity

to obtain

$$
t^{m}=\sum_{k=0}^{m} \mathscr{S}_{m}^{(k)} t(t-1) \ldots(t-k+1)
$$

Now, for $\xi \in X$,

$$
\hat{\varrho}_{n}\left(\Lambda_{1}^{m_{1}} \ldots \Lambda_{r}^{m_{r}}\right)=\int_{X} d \mu\left(\# \xi_{A_{1}}\right)^{m_{1}} \ldots\left(\# \xi_{A_{r}}\right)^{m_{r}} .
$$

$$
\left(\# \xi_{A_{1}}\right)^{m_{1}} \ldots\left(\# \xi_{A_{r}}\right)^{m_{r}}
$$

is the number of ways one can choose ordered $n$-tuples of integers

such that

$$
\left(i_{1}, i_{2}, \ldots, i_{n}\right)
$$

$$
\left(x_{i_{1}}, x_{i_{2}}, \ldots, x_{i_{n}}\right) \in \Lambda_{1}^{m_{1}} \ldots \Lambda_{r}^{m_{r}} .
$$

Notice that we do not demand that

$$
i_{k} \neq i_{j} \quad \text { if } \quad k \neq j .
$$

This combinatoric fact shows us what our definition of $\hat{N}_{H}$ must be for arbitrary $H \in \mathscr{R}^{n}$ : for

and $H \in \mathscr{R}^{n}$

$$
\xi=\left(x_{1}, x_{2}, \ldots\right) \in X
$$

$\hat{N}_{H}(\xi):=$ The number of ways one can choose ordered $n$-tuples of integers

such that

$$
\left(i_{1}, \ldots, i_{n}\right)
$$

$$
\left(x_{i_{1}}, \ldots, x_{i_{n}}\right) \in H \text {. }
$$

\section{Appendix 2: On The Classical Moment Problem}

Suppose we are given a sequence of real numbers

$$
\left(S_{k}\right)_{k=0}^{\infty}\left[S_{0}=1\right] \text {. }
$$

The Hamburger moment problem is concerned with the existence and/or uniqueness of a real valued non-decreasing function $\sigma$ satisfying

$$
S_{k}=\int_{-\infty}^{\infty} \lambda^{k} d \sigma(\lambda) \quad k=0,1,2, \ldots
$$


In the above expression the integral is that defined by the LebesqueStieltjes measure determined by $\sigma$. In the following we shall let $\sigma$ denote either the non-decreasing function or the measure associated with it. The Stieltjes moment problem is concerned with non-decreasing functions satisfying

$$
S_{k}=\int_{0}^{\infty} \lambda^{k} d \sigma(\lambda) \quad k=0,1,2, \ldots
$$

The moment problem is said to be determinate if the solution exists and is unique in the sense that it determines the same measure on $\mathbb{R}$. The following theorem is due to T. Carleman (Wall, Ref. [4]).

Theorem A.2-A. a) Let $\left(S_{k}\right)_{k=0}^{\infty}$ be a sequence of real numbers for which a solution of the Hamburger moment problem exists. This moment problem is determinate if

$$
\sum_{k=0}^{\infty}\left|S_{2 k}\right|^{-\frac{1}{2 k}}=\infty
$$

b) Let $\left(S_{k}\right)_{k=0}^{\infty}$ be a sequence of real numbers for which a solution of the Stieltjes moment problem exists. This moment problem is determinate if

$$
\sum_{k=0}^{\infty}\left|S_{k}\right|^{-\frac{1}{2 k}}=\infty .
$$

Consider $\mathscr{L}_{2}(\sigma)$ where $\sigma$ is the solution of some moment problem. Since the moments are, by definition, finite, it is obvious that the polynomials are in $\mathscr{L}_{2}(\sigma)$. A natural problem that arises is to find necessary and sufficient conditions on $\sigma$ to ensure that the polynomials are dense in $\mathscr{L}_{2}(\sigma)$. Before stating the theorem, due to Naimark, which characterizes those measures for which the polynomials are dense we point out that the solutions of a given moment problem form a convex set of measures. The extreme points of this set are called the extremal solutions ${ }^{1}$ of the moment problem.

Theorem A.2-B (Naimark). Let $\sigma$ be a Stieltjes measure on $\mathbb{R}$. The polynomials are dense in $\mathscr{L}_{2}(\sigma)$ if and only if $\sigma$ is an extremal solution of some Hamburger moment problem.

The following proposition tells us that determinate solutions of Stieltjes moment problems satisfy this condition.

Proposition A.2-1. Let $\left(S_{k}\right)_{k=0}^{\infty}$ be a sequence of real numbers for which $\sigma$ is a determinate solution of the Stieltjes moment problem. Then $\sigma$ is an extremal solution of the corresponding Hamburger moment problem.

\footnotetext{
1 Akheizer (Ref. [5]) calls these solutions $V$-extremal to distinguish them from solutions that he terms $N$-extremal.
} 
Proof. First we point out that be defining:

$$
\sigma(\lambda):=\sigma(0) \quad \text { for } \quad \lambda<0
$$

we extend $\sigma$ to be a non-decreasing function on $\mathbb{R}$ and hence define a Stieltjes measure on $\mathbb{R}$ which is a solution of the Hamburger moment problem. Suppose that $\sigma$ is not an extremal solution. Then there exists $\sigma_{1}$ and $\sigma_{2}$, non decreasing functions, which determine different solutions of the Hamburger moment problem and satisfy

$$
\sigma=\alpha \sigma_{1}+(1-\alpha) \sigma_{2}
$$

for some $\alpha \in(0,1)$. This equality holds in the sense of Stieltjes measures on $\mathbb{R}$.

Since $\sigma$ vanishes on $(-\infty, 0]$ it follows that both $\sigma_{1}$ and $\sigma_{2}$ must vanish on $(-\infty, 0]$. Thus the supports of $\sigma_{1}$ and $\sigma_{2}$ are contained in $[0, \infty)$ and clearly than $\sigma_{1}$ and $\sigma_{2}$ are solutions of the Stieltjes moment problem. By assumption this problem is determinate and hence $\sigma_{1} \neq \sigma_{2}$ cannot exist.

Acknowledgement. The author would like to acknowledge his gratitude and appreciation to A. Lenard for the original suggestion of the problem and the many helpful suggestions which culminated in the doctoral dissertation upon which this paper is based. $\mathrm{He}$ also would like to express his thanks to Mrs. Kathy Sams for a swift and sure job of typing.

\section{References}

1. Ruelle, D.: Math. Phys. 8, 1657-1668 (1967)

2. Lenard, A.: Commun. math. Phys. 30, 35-44 (1973)

3. Masson, D., McClary, W. K.: J. Funct. Anal. 10, 19-32 (1972)

4. Wall, H.S.: Analytic theory of continued fractions. New York: D. Van Nostrand Co. 1948

5. Arkiezer, N.I.: The classical moment problem and some related questions in analysis. Edinburgh: Oliver and Boyd 1965

Communicated by H. Araki

\author{
Sanford I. Levy \\ Department of Mathematics \\ Indiana University \\ Bloomington, Indiana 47401, USA
}

